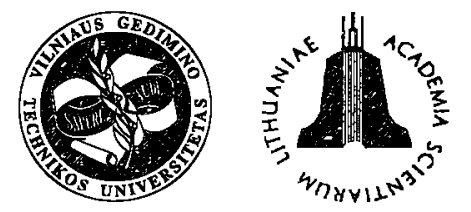

ISSN 1648-4142 TRANSPORT

http:/www.vtu.lt/english/editions

TRANSPORT - 2003, Vol XVIII, No 3, 108-113

\title{
THE SOLUTION OF CUSTOMS POSTS MANAGEMENT PROBLEMS AT RAILWAY BORDER STATIONS
}

\author{
Aldona Jarašūnienè \\ Transport Research Institute, Vilnius Gediminas Technical University, Plytinès g. 27, LT-2040 Vilnius, Lithuania \\ E-mail: ajarasuniene@yahoo.com
}

Received 20021211 ; accepted 20030430

\begin{abstract}
The Identification and solution of transportation problems related to customs posts at railway border stations enable successful functioning of international freight transport, thus guaranteeing its reliability, punctuality and high quality. Perfection of the complex work is necessary in order to meet the requirements as well as to preserve goods during their transportation process. For the solution of the problems at the stations it is important to analyse fundamental principles, to assess an environmental impact, to formulate and evaluate the issues of wagon management by means of complicated technical systems, and to optimise the process of problems on the basis of rescarch methods.
\end{abstract}

Keywords: identification of transportation problems; optimisation of their process; mathematical modelling.

\section{Introduction}

With the growth of international freight transportation by rail the main attention should be focused on the functions of railway border stations, where the arriving trains are disbanded and new trains are arranged according to the train formation plan. Such operations as the formation of incoming, outgoing and transit goods, freight admission, export, redistribution, freight preservation, storage, clearance of freight and wagon documentation are going on. It is at these stations that customs formalities are proceeded, based on the identification of transportation problems, on the optimisation of ongoing operations and customs process management technologies. This allows successful functioning of international freight transportation, thus guaranteeing its reliability, punctuality and high quality.

The efficiency of international freight transportation should depend on the optimisation of the processes of customs formalities.

During the train survey at the customs posts at railway border stations the correction work related to seal infraction, broadside reinforcement, bolt fastening, necessary for placing and fastening of goods on a mobile train is carried out.

At present reorganisation activities are going on at customs posts at railway border stations, which make a positive influence on the survey of wagons. The quality of survey makes a positive influence on the safety and preservation of transported goods and their efficient delivery to clients. However it is not sufficient [1-7].
The perfection of complex work is necessary in order to meet the requirements and optimally to preserve goods in the process of transportation.

For the solution of transportation problems occurring at the customs posts at railway border stations the main objective is to identify the reasons of their occurrence, their basic principles, the assessment of environmental impact, the formulation and evaluation of tasks for wagon management by complex technical systems, the optimisation of problems process, based on the scientific research methods [8].

\section{The Theory and Analysis of Management by Com- plex Technical Systems}

Principles of the theory of management. For the analysis of the origin of transportation problems at customs posts at railway border stations it is necessary to identify the basic principles of the occurrence of the problems and to define their environmental impact.

For the evaluation of transportation problems of customs posts at railway border stations it is necessary to gauge their volume, also the impact of various preconditions causing their occurrence, their structure and groups.

Transportation problems are the problems arising from unsafe transportation of freight, inadequate sorting, handling, filling in the documentation, sealing, and low quality delivery to the consignees.

For the assorting of transportation problems it is necessary to evaluate the following criteria:

1. the criterion of physical state; 
2. the criterion of the method of wagon loading;

3. the criterion of possibility of generalisation of transportation problems.

In the course of the analysis of the origin of transportation problems at customs posts at railway border stations it is possible to find the ways of their elimination.

This analysis is based on the creation of the evaluation algorithm [9].

The scope of transport problems comprises the prime cost analysis, which has been vastly used by Taylor for analysing problems of this type. Thus named Taylor's system is determined by the costs of arrangement of operation methods and management of production processes (by the way of selection and organisation of labour force improving the standards of activities) [10].

Analysis of management of complex technical systems. Management problems are very acute. Based on scientific research and complex approach the solution and versatility of wagon management by complex technical systems are peculiar to railway border stations.

Wagon management by complex technical systems is improved basing on various measures.

However, a theory of management of customs posts at railway border stations does not exist. Also the problems and their solutions in the management of customs control facilities are not identified.

To start the solution of these issues great efforts are necessary as well as an indispensable unified programme comprising all the work starting with projecting to the practical realisation. It is important to apply the mathematical calculation methods that would enable the identification of technical capacities of customs control posts at railway border stations, which would allow the growth of international freight volumes.

Under real conditions the qualitative formulation of a production and operational process of complex technical systems is influenced by various factors. Deficiencies of theoretical research make a signifficant share of these factors influencing the quality of complex technical systems.

For the determination of the quality standards it is necessary to gauge them. Positive results in management field make a positive influence on the stability and rhythm of a transportation process, the accurateness of technological discipline and the implementation of organisation of scientific research activities.

The management of the quality of complex technical systems in correspondence to the general theory of the station management presents a uniform versatile process and consists of the following operations: analysis of management programme, planning of transportation quality improvement (transportation safety, transported goods safety/preservation, handling of documentation transported), obtaining and analysing of the information on the state of objects participating in the transportation process.

The quality management of a complex technology system corresponds to the general theory of station management and embodies a uniform multiplan process consisting of the following operations:

- creation of management programmes;

- planning of the improvement of transportation quality (transportation safety, freight safety, documenttation handling, etc. );

- obtaining and analysing of the data about all the hauliers participating in freight transportation;

- giving orders and analysing of the information obtained.

Thus the management or technology of complex technical system quality is the implementation, service and generalising of measures. The main principles of technology management are: sistematics/organisation, identification of tasks, adaptation, dynamics, quality norms, standardisation.

Optimal attainments of technology quality standards are identified on the basis of cost price of organisationaltechnical and economical measures which are commercially interrelated and have an influence on corresponding factors and conditions. The creation of the model of customs posts at railway border stations starts with the analysis of the modelling subject by the application of mathematical formulae and generation of information. This is done with the aim of qualitative co-ordination in an experimental way and the fulfilment of a task, as well as for the analysis, correction of the model by additional solutions and eventually, to make the final verification of the model. Only after these operations the model may be transferred to information system for use and implementation of the tasks [9].

The analysis of the subject must give a full view of the system modelled and its modelling possibilities.

The mathematical system formulation will determine the future efficiency of the system. The mathematical formulation of the system is the modelling of the entire process, i.e. the description of economical processes and mathematical-economical actions of the model. The aim of modelling is the possibility to manage and control a concrete process [10].

Several mathematical methods are used for the organisation of the process:

- Optimal programming: linear, non-linear, discrete, block, etc.;

- Network methods of management and planning;

- Theory of mass service, etc.

Mathematical model may be generally expressed as follows:

$$
\begin{gathered}
z=f\left(x_{1}, x_{2}, \ldots, x_{n}\right) \rightarrow \max (\min ) \\
\varphi_{i}=\left(x_{1}, x_{2}, \ldots, x_{n}\right) \leq b_{i}\left(i=1,2, \ldots, m_{1}\right) ; \\
\varphi_{i}=\left(x_{1}, x_{2}, \ldots, x_{n}\right)=b_{i}\left(i=m+1, m_{1}+2, \ldots, m_{2}\right) ;
\end{gathered}
$$




$$
\begin{aligned}
& \varphi_{i}=\left(x_{1}, x_{2}, \ldots, x_{n}\right) \geq b_{i} \quad\left(i=m_{2}+1, m_{2}+2, \ldots, m\right) \\
& x_{j} \geq 0 \quad\left(j=1,2, \ldots, n_{1}\right) \\
& x_{j} \quad\left(j=n_{1}+1, n_{2}+2, \ldots\right) \\
& x_{j} \leq 0 \quad\left(j=n_{2}+1, n_{2}+2, \ldots, n\right)
\end{aligned}
$$

where (1) is the aim function; (2)-(7) - system of limitations; $b_{i} \geq$ free members of the limitations $(l=1,2, \ldots, m)$.

The purpose of the system is to demonstrate the system condition, which should be reached in the process of management. The application of the methodological foundation for the creation of technological management would be able to satisfy all the market needs and requirements.

The efficiency of the technological process is evaluated by the only economical criteria - the national income growth in respect of production costs or resources used for production under the conditions of the optimal proportion of consumption and accumulation funds.

All national economical criteria by their content may be attributed to one of the three types:

- to the maximum economic effect under fixed costs;

- to the minimum costs under fixed effect;

- to the maximum economic effect with the use of available resources.

Given a concrete task the economic criteria should correspond to the following requirements:

- to reflect objective national needs for service system;

- to reflect the necessity of this service for the national economical system;

- to express costs and results;

- to forecast the scientific progress of services of this field.

Quite often the selection of the optimal criterion causes certain difficulties and problems, which can not be solved unambiguously. The issue of a complex technical system criterion most often undergoes versatile analysis so that to avoid future errors of the solution of the problems. Most often the criteria depend on the management parameters $\left(x_{j}\right)$. The variable value changes depend on the quantity of variation possibilities. Most characteristic ones are in the tasks carried out by the application of various technologies. Often not only corresponding technologies are included into the model, but indices defining the parameters of the system economical parameters as well.

\section{The Ways of Solution of Transportation Problems on the Basis of Time Definition Analysis}

Solving transport problems technical capacities of customs posts at railway border stations may be defined basing on mathematical calculation methods.

For the investigation of the interface between the sub-system "Arrival ways - mechanised ways" and the evaluation of the system at command functioning reliability, we will apply the method of mass service with regard to characteristics of stochastic elements in the process of elimination of problems at customs posts at railway border stations.

Aiming at the elimination of transportation problems we shall analyse the technology of a one-channel system. In general, the time devoted to the solution of the problem may be distributed into the time $\left(t_{1}\right)$ for waiting for the unoccupation of the channel-aggregate and the time $\left(t_{2}\right)$ for the elimination of the problem. In this case the time of problem solving will be considered as random and distributed on the exponential basis.

For the selection of the parameters $\left(t_{1}\right)$ and $\left(t_{2}\right)$ we shall use a well-known Laplace's distribution variation function with regard to the priorities determining that a train consisting of covered wagons will be considered prioritive and the train consisting of non-covered wagons - non-prioritive.

$$
G_{(2)}^{x}(S)=\frac{H_{2}^{x}\left(S+\lambda_{1}\right)}{1-\frac{\lambda_{1}}{\lambda_{1}+S} \cdot \psi_{1}^{x}(S) \cdot\left[1-H_{2}^{x}(S+\lambda)\right.}
$$

where $\lambda_{1}$ - the intensity of prioritive transportation problems (in covered wagons); $\lambda_{2}$ - the intensity of usual transportation problems (in open, non-covered wagons); $\psi_{1}^{x}(S)$-the variation of the Laplace distribution function in the channel occupancy interval, under the prioritive model (for instance, covered or other type wagons bearing the witness of plunder); $H_{2}^{x}(S)$ - the time of duration of variation of distribution of Laplace's function in the case of non-prioritive transportation problems (under service/ handling).

Let us define the time duration $\left(\mu_{2}\right)$ of prioritive order $\left(\mu_{1}\right)$ and of other orders as well. Thus

$$
\psi_{1}^{x}(S)=\frac{\mu_{1}}{\mu_{1}+S} ; H_{2}^{x}(S)=\frac{\mu_{2}}{\mu_{2}+S} .
$$

Putting the significance (9) into the formula (8), we shall obtain the formula:

$$
G_{2}^{x}(s)=\begin{gathered}
\mu_{2}\left(S+\mu_{1}\right) \\
\left(S+\mu_{1}\right)\left(S+\lambda_{1}+\mu_{1}\right)-\lambda_{1} \cdot \mu_{1}
\end{gathered} .
$$

For the determination of the time of mathematical expectancy $\ddot{t}_{2}$ and dispersion $S\left(t_{2}\right)$ in the elimination of non-prioritive commercial problems we shall apply the features of the characteristics of the function.

It is known that the significance of the constituent $k$-th equals to the initial definition moment $G_{2}^{x}(S)$. Differentating the $G_{2}^{x}(S)$ in the formula (8) according 
to $S$ and assuming that $S=0$, we shall determine the time destined for the elimination of commercial problems, the mathematical expectancy $t$ by the formula $t_{2}=d G_{2}^{x}(S)$. When $S=0$, we shall obtain the formula: $\mu_{1}+\lambda_{1}$. $\mu_{1} \cdot \mu_{2}$

The dispersion of the service/handling time $S\left(t_{2}\right)$ will be determined by the second derivative from $G_{2}^{x}(S=0)$, when $S=0$.

The determination of time duration of commercial problems service/handing is analogical.

The determination function for time duration of settled work regime is as follows:

$$
V_{2}^{x}(S)=\frac{P_{0}^{2}\left[S+\lambda_{1}\left(1-\psi_{1}^{x}(S)\right]\right.}{S-\lambda_{2}\left[1-G_{2}^{x}(s)\right],}
$$

where $P_{0}^{2}$ is the expectancy of being in a free position.

If the work lasts only 8 hours per day/per 24 hours, then the significance for service/handling discipline is:

$$
P_{0}^{2}=1-\frac{\lambda}{\mu_{1}}-\frac{\lambda_{2}}{\mu_{2}}\left[1-H_{2}^{x-1}(\lambda)\right] .
$$

When the time period between the start and finish of service/handling has positive distribution, we shall determine the significance:

$$
P_{0}^{(2)}=1-\frac{\lambda^{1}}{\mu_{1}}-\frac{\lambda_{2}}{\mu_{2}} .
$$

Based on the formula (8), the significance of waiting time for channel unoccupation will be as follows:

$$
V_{2}^{x}(S)=\frac{\left(1-\frac{\lambda_{1}}{\mu_{2}}-\frac{\lambda_{2}}{\mu_{2}}\right)\left(S+\frac{S \lambda_{1}}{\mu_{1}+S}-1\right)}{S-\frac{\lambda_{2}: \mu_{2}\left(S+\mu_{1}\right)}{\left(S+\mu_{1}\right)\left(S+\lambda_{1}+\mu_{2}\right)-\lambda_{1} \mu_{1}}} .
$$

Differentiating $V_{2}^{x}(\bar{S})$ in line with $S$, we will find the average time significance for the elimination of commercial problems:

$$
\bar{t}_{1}=\frac{\lambda_{2} \bar{t}_{2}^{2}}{2\left(1-\lambda_{2} \bar{t}_{2}\right)}-\frac{\lambda \cdot \bar{\tau}_{2}}{2\left(1+\lambda_{1} \tau_{1}\right)}
$$

where $\tau_{1}$ and $\bar{\tau}_{1}^{2}$ mean the initial time moments of service/handling of the first and the second prioritive orders. These significances will be defined by the following formulae:

$$
\bar{\tau}_{1}=H_{1}^{\prime x}(S) / S=0=\frac{1}{\mu_{1}} ;
$$

$$
\tau_{2}=H_{1}^{n x}(S) / S=0=2\left(\begin{array}{c}
1 \\
\mu_{1}
\end{array}\right)^{2} .
$$

Given that the inetrvals between the mending of transportation problems are determined by the exponential distribution, it is possible to apply $\gamma-$ for deeper detalisation.

The channel is analysed, which has no interference - the production costs almost cannot be eliminated.

Thus we shall determine the mathematical duration and the dispersion of two general intervals of indicated priorities. Then the formula for the mathematical expectancy of the general interval will be:

$$
t=\frac{\lambda_{0} / \mu_{1}+\lambda_{1} / \lambda_{0}\left(1-\frac{1}{H_{1}^{x}\left(\lambda_{0}\right)}\right)}{\left(\lambda_{0}-\lambda_{1}\right) \cdot\left[1-\frac{\lambda_{0}}{\mu_{1}}-\frac{\lambda_{1}}{\lambda_{0}}\left(1-\frac{1}{H_{1}^{x}\left(\lambda_{0}\right)}\right)\right.} .
$$

Assuming that the interference occurs because of the first level priorities and assuming the condition that $\lambda_{0}=\lambda_{1}$, we shall obtain:

$$
t_{1}=\frac{\lambda_{1} / \mu_{1}+1-1 / 1+\lambda_{1} / \mu_{1}}{2 \lambda_{1}\left[1-\frac{\lambda_{1}}{\mu_{1}}+\frac{1}{1+\lambda_{1} / \mu_{1}}\right]} .
$$

Given the general duration of the interference interval and that of the first level priority, it is possible to determine the mathematical expectancy time of transportation problems.

Flows of two types exist in the channel: prioritive and non-prioritive. Non-prioritive is the flow of open type wagons orders, prioritive is the general flow of orders of covered wagons train and interferences. Then:

$$
\begin{aligned}
& t_{2}=\left(\begin{array}{c}
1-H_{2}^{x}\left(\lambda_{0,1}\right) \\
+t_{1}
\end{array}\right) \\
& t_{1}=\frac{\lambda_{2} t_{2}^{2}}{2\left(1-\lambda_{2} t_{2}\right)}-\frac{\lambda_{0,1} t_{1}}{2\left(1+\lambda_{0,1} t_{1}\right)}
\end{aligned}
$$

Thus, after performing calculations we obtain $t_{b e n d r .}=t_{1}+t_{2}$, which is important for the determination of the technical capacities of customs posts at the railway border stations.

\section{Management of Transport Flows at Railway Bor- der Stations}

Making the analysis of management of transport flows at railway border stations it is presumed that trans- 
port flows are moving in the given network $G=(V, E)$ which has $n$ peaks and $m$ panes. In the network the beginning of flow at the peak 1 and the end of flow at the peak $n$ are fixed. The traffic of the flow along each pane lasts one time interval. The time is discrete and has only positive meanings $t=0,1, \ldots, T$, here $T$ - period of management (planning) [11-12].

At each time moment $t$ the state of the process of movement of flow is characterised by $n$ phasic coordinates $x_{1}(t), \ldots, x_{n}(t)$, here $x_{i}(t)$ - the amount of the flow remaining on the peak $i$ at the time interval $[t, t+1]$. Thus at every moment $\mathrm{t}$ the phasic state $X(t)$ has $n$ coordinates:

$$
X(t)=\left(x_{1}(t), x_{2}(t), \ldots, x_{n}(t)\right) .
$$

The object state sequence $X(0), X(1), \ldots, X(T)$ at the moments $t=0,1, \ldots, T$ is called the trajectory of the movement of the object.

We will manage the flow movement at each time $t$ moment selecting $m$ managing parameters $U(t)=\left(u_{1}(t), \ldots, u_{m}(t)\right.$, here $u_{j}(t)$ - amount of flow starting to move along the pane $j$ at $t$ time moment.

The sequence of points is called the management $U(1), U(2), \ldots, U(T)$. We introduce restrictions to management field and to phasic coordinates.

It is considered that the possibilities of the pane capacity and capacity of stores at the peaks are the functions of the time $t$ and incidental factor $\xi$. Let us indicate:

$$
f(t, \xi)=\left(f_{1}(t, \xi), \ldots, f_{m}(t, \xi)\right)
$$

and

$$
l(t, \xi)=\left(l_{1}(t, \xi), \ldots, l_{m}(t, \xi)\right) .
$$

Accordingly the vectors of capacity possibilities and the lower margin at the moment $t$, specify a permissible management field, and

$$
g(t, \xi)=\left(g_{1}(t, \xi), \ldots, g_{n}(t, \xi)\right)
$$

store capacity vector specifies restrictions to phasic coordinates.

Then, in the network of transport flows, we shall formulate the optimal management issue as the stochastic issue of optimal management of a discrete object:

$$
\begin{aligned}
& J_{0}(X, U, t) \rightarrow \max \text {; } \\
& \Delta X(t)=\varphi(U, t), \quad t=\overline{0, T-2} ; \\
& l(t, \xi) \leq U(t) \leq f(t, \xi), \quad t=\overline{0, T-1} ; \\
& X(T) \leq g(t, \xi), t=\overline{1, T} ; \\
& \left.\begin{array}{l}
X(t) \geq 0 \\
U(t) \geq 0
\end{array}\right\}, \quad t=\overline{0, T} .
\end{aligned}
$$

And the set of initial states $X(0)$ - is put:

$$
X(0)=X^{0},
$$

here (22) - criterion of management efficiency;

(23) - management of the movement of a managed object;

(24) - permissible management range;

(25) - limitations for phasic coordinates;

(26) - non-negativity of phasic variable and managing parameters.

By the stochastic model (22)-(26) it is possible to define a great class of transport flows optimal management objectives.

Let $\beta b_{i j}$ be the transport network $G$ matrix of peakpane incidences, whereas $b_{i j}=-1$, if the pane $j$ goes out from the peak $i$ and $b_{i j}=+1$, if the pane $j$ goes into the peak $i$. The matrix $\beta^{+}$is obtained also changing the negative elements of $\beta$ by zeroes, and $\beta$ - changing positive elements with zeroes. Then discrete managed object which is defined by the conditions (22)-(26) will be described in the network of dynamic flow in proportion to the respective parameters:

$$
\begin{aligned}
& X(t+1)=X(t)+B^{+} U(t)+B^{-} U(t+1), t=0, T-2 \\
& X(T)=X(T-1)+B^{+} U(T-1) \\
& X(0)=X^{0} \\
& l(t, \xi) \leq U(t) \leq f(t, \xi) \\
& X(t) \leq g(t, \xi), t=\overline{1, T} \\
& \left.\begin{array}{l}
0 \leq X(t) \\
0 \leq U(t)
\end{array}\right\}, \quad t=0, T .
\end{aligned}
$$

The theoretical research of transport flows at railway border stations allows to make a detailed analysis of freight flows enabling the elaboration of the recommendations for the improvement of customs inspection procedures. Basing on this the analysis of freight structure and distribution according to countries would enable to prepare the most influential forecasts of transit freight transport by separate customs-houses.

\section{Conclusions}

1. Aiming at the optimal use of opportunities for international railway transportation it is necessary to analyse the problems of customs posts at railway border stations.

2. The solution of the problems has to be based on the consideration of the reasons of the occurrence of these problems, together the environmental impact of the optimisation of transportation process problems on the basis of scientific research work has to be evaluated.

3 . The creation of the model of customs posts at the railway border stations and such operations as the analy- 
sis of the modelled object, mathematical-economical acts of the model, the accumulation of information, the efficiency of model verification - the performance of all these operations would enable successful management and control of in-coming, out-going and transit freight flows.

4. The mathematical calculation on the basis of the theory of mass service/handling would enable the determination of the technical capacities of customs posts at railway border stations.

5. Further, basing on theoretical research, it would be possible to make the analysis of the distribution (according to time) of flows of cars entering a customs post as well as the analysis of their distribution into separate customs lanes. It would also be possible to identify the idle time of transport means caused by customs procedures, to identify the dependence of the time of services given to carriers in proportion to the amount of transport flow, to calculate and to assess the optimal selection of transport amount for inspection at customs during a certain period of time.

\section{References}

1. Southworth, Frank; Peterson, Bruce E. Intermodal and intermodal freight network modelling. Transportation $R e$ search, Part C 8, 2000, p. 147-166.

2. Warren, Powell B.; Tassio Carvalko. A Real-Time Optimisation of Containers and Flatcars for Intermodal Operations. Transportations Science, Vol 32, No 2, May 1988 , p. $110-126$.

3. Zhan, F.-B.; Noon, Ch.-E. Shortest Path Algorithms: An
Evaluation Using Real Road Networks. Transportation Science, Vol 32, No 1, February, 1988, p. 65-73.

4. Nuzzolo, A.; Russo, F.; Cristali, U. A Doubly Dynamic Schedule - Based Assignment Model for Transit Networks. Transportation Science, Vol 35, No 3, August, 2001, p. 268285

5. Gattorna, D.; Walters, W. Managing the Supply Chain. A Strategic Perspective. Macmillas Press Ltd., 1998. 310 p.

6. Coule, J.; Bordi, E.; Carinato, J. Transportations. Third edition. USA, 1999. $525 \mathrm{p}$.

7. Transport of Fast Changing Europe. Vers un Reseau European des systems de transport by Group Transport 2000 Plus. Brussels, 2000. 90 p.

8. Baublys, A. Introduction to the theory of transport system (Transporto sistemos teorijos ivadas). Vilnius: Technika, 1997. 298 p. (in Lithuanian).

9. Grau, B. Typical technological process of work by distribution station (Типовой технологический процесс работы сортировочной станции). Moscow: Transport, 1995. 50 p. (in Russian).

10. Sotnikov E. Elements of managing theory by system of quality (Элементы теории управления качеством системы). Minsk: Nauka i technika, 2001. 41 p. (in Russian).

11. Jarašūnienè, A. The optimization of transport flows crossing Lithuanian customs-houses. Transport, Vol XVII, No 1, Vilnius: Technika, 2002, p. 15-18.

12. Jarašūnienè, A. Formulation of optimal international freight transport objective. Transport, Vol XVII, No 5, Vilnius: Technika, 2002, p. 201-204. 